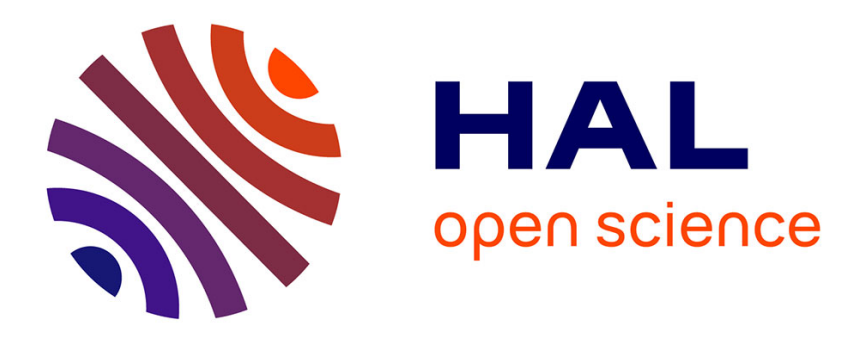

\title{
Artus de Bretagne Un roman de la jeunesse et de la désinvolture
}

\author{
Sébastien Douchet, Valérie Naudet
}

\section{To cite this version:}

Sébastien Douchet, Valérie Naudet. Artus de Bretagne Un roman de la jeunesse et de la désinvolture. Christine Ferlampin-Acher. Artus de Bretagne. Du manuscrit à l'imprimé (XIVe-XIXe siècle), Presses Universitaires de Rennes, 2015. hal-01441645

\section{HAL Id: hal-01441645 https://hal-amu.archives-ouvertes.fr/hal-01441645}

Submitted on 20 Jan 2017

HAL is a multi-disciplinary open access archive for the deposit and dissemination of scientific research documents, whether they are published or not. The documents may come from teaching and research institutions in France or abroad, or from public or private research centers.
L'archive ouverte pluridisciplinaire HAL, est destinée au dépôt et à la diffusion de documents scientifiques de niveau recherche, publiés ou non, émanant des établissements d'enseignement et de recherche français ou étrangers, des laboratoires publics ou privés. 


\title{
Artus de Bretagne \\ Un roman de la jeunesse et de la désinvolture
}

\author{
Sébastien Douchet et Valérie Naudet \\ Université d'Aix-Marseille (CIELAM/CUER MA)
}

«Hector, ce dist Artus, nous sommes jones $! »^{1}$. Cette exhortation enthousiaste introduit dans Artus de Bretagne une notion centrale pour sa compréhension. Artus, Florence, Hector et bien d'autres personnages sont qualifiés de jones du début à la fin de ce roman qui est sans conteste un roman de la jeunesse ${ }^{2}$, plus encore qu'Érec et Énide ou que le Conte du graal qui lançaient des jeunes en aventure, et davantage que les romans arthuriens de la seconde génération qui inventaient des fils à ceux qui furent les jeunes héros de la première génération.

Dans Artus de Bretagne, les chevaliers ne sont plus preux et vaillants, mais « gentil et noble et jone ", les demoiselles ne sont plus belles et courtoises mais " douce et gracieuse et jone » ou même « jone et tendre ». C'est, nous dit le texte, " par jonesce » qu'Artus et ses compagnons sont partis en aventure $^{3}$. La jeunesse et sa fougue sont le moteur qui lance l'action romanesque et non la beauté éblouissante de chevaliers rencontrés dans une forêt ou encore la honte de la recreance. La jeunesse, pas les armes et l'amour.

Cette énergie juvénile est au cœur du roman. Elle en est même est la pièce maîtresse : elle affecte la façon dont sont construits les personnages. Elle permet aussi de mieux comprendre le recyclage de la matière ancienne, de son écriture et de ses enjeux. La jeunesse des personnages est inséparable d'une forme de rajeunissement du roman qui s'opère non sans une certaine désinvolture, c'est-à-dire avec légèreté et sans grande considération pour un héritage littéraire dont le sens et les fonctions premières sont intentionnellement ignorées. Le bain de jouvence que fait subir Artus de Bretagne à ses vieux prédécesseurs a parfois été radical.

La lecture de l'épisode initial du roman sert de point de départ à notre étude. Une longue entrée en matière ouvre le roman qui prend immédiatement ses distances avec les textes antérieurs qui constituent son héritage et son socle référentiel. Cela se traduit par un recyclage et une neutralisation de la matière ancienne sur un plan aussi bien textuel que matériel comme le montrent le traitement de quelques motifs et de certains traits d'écritures, mais aussi l'organisation matérielle et l'iconographie du manuscrit de Paris, le plus ancien de tous.

\section{Les anciens tous derrière, et les jeunes devant...}

Le roman s'ouvre de façon curieuse sur une quarantaine de paragraphes dont le caractère problématique et la difficile insertion dans l'économie globale de l'œuvre ont été soulignés ${ }^{4}$. Cet incipit entrelace le récit des enfances d'Artus, sa rencontre avec Jehanette et son mariage avec Péronne d'Autriche, au récit de la jeunesse de Florence, installant ainsi le roman dans son double univers breton et oriental. Mais ce faisant, l'incipit dépasse largement son rôle liminaire et finit, à force de zèle, par ne plus remplir sa fonction de seuil de l'œuvre.

1 Artus de Bretagne, f. $5 \mathrm{v}^{\circ} \mathrm{a} ; \S 14,1.11$. Nos références renvoient au BnF français 761 (voir Gallica http://gallica.bnf.fr/ark:/12148/btv1b84478715/f1.image, page consultée le 28 mai 2014) et à l'édition de Ch. FERLAMPIN-ACHER, Artus de Bretagne, Paris, Champion, CFMA, à paraître. Nous tenons à exprimer toute notre gratitude à Christine FERLAMPIN-ACHER pour nous avoir communiqué son texte.

2 «...Li dui enfant s'esjoïssoient en grant leece de cuer pour le dous temps, comme cil qui estoient jone et a qui il ne falloit fors que jouer et rire... » (Ibid., f. $3 \mathrm{r}^{\circ} \mathrm{c}-3 \mathrm{v}^{\circ} \mathrm{a} ; \S 7,1.20-22$ ).

Ibid., f. $4 \mathrm{r}^{\circ} \mathrm{b} ; \S 10,1.15-16 ;$ f. $25 \mathrm{r}^{\circ} \mathrm{c} ; \S 55,1.7$; f. $91 \mathrm{v}^{\circ} \mathrm{b} ; \S 296.1 .9$; f. $26 \mathrm{r}^{\circ} \mathrm{a} ; \S 57,1.5$.

4 FERLAMPIN-ACHER Ch., «Artus de Bretagne : une histoire sans fin », Clore le récit II, Pris-ma XV/1, p. 56-57. 
Le problème vient du fait que le roman commence pour ainsi dire quatre fois, faisant signe vers des horizons intertextuels génériquement divers. Le texte débute en effet comme un roman biographique avec les enfances d'Artus ${ }^{5}$. Mais quittant rapidement ce sujet, une seconde séquence s'ouvre à la façon d'un lai féerique et narre les mésaventures de Jehanette ${ }^{6}$, jeune fille avatar de fée rencontrée au bord d'un étang au cours d'une chasse au cerf au plus profond d'une épaisse forêt, et dont s'éprend Artus. Mais à peine l'aventure amoureuse s'est-elle nouée qu'on la quitte pour un troisième volet qui relève du roman matrimonial consacré au mariage forcé $d$ 'Artus avec Péronne . Après l'échec cuisant de cette mascarade nuptiale, on délaisse l'univers breton, ses lacs et ses demoiselles, pour se tourner vers l'Orient et le royaume d'Emenidus, "entre Perse et Mesopotame $\gg{ }^{8}$. Cette séquence de roman oriental conte les enfances de Florence et d'Estienne et présente les merveilles de la Porte Noire ${ }^{9}$, alors qu'entre temps un roman d'aventure semble avoir débuté avec le départ d'Artus, Hector et Gouvernal pour le vaste monde ${ }^{10}$. Quelle est la fonction de cette ouverture complexe et multiple ? Pourquoi lancer tant de pistes au risque d'égarer dès le départ un lecteur dérouté par une telle profusion?

En fait, ce n'est pas tant la multiplication de ces seuils rhétoriques qui semble intéresser l'auteur, même si elle lui offre une (première) occasion de démontrer une certaine virtuosité d'écriture, que la possibilité de mettre plusieurs fois sur le devant de la scène un thème, celui de la jeunesse. Plutôt qu'un entrelacement des aventures, c'est un entrelacement des jeunesses des héros qui unit ces premiers paragraphes. Cette époque de la vie s'affirme d'emblée comme cruciale, car ces seuils successifs font entrer le lecteur dans l'œuvre par des récits de jeunesse, laquelle est l'une des clefs de lecture du roman.

Cette jeunesse est revendiquée par les personnages pour justifier leurs actes : à son cousin Hector auquel son père vient sèchement d'apprendre qu'il ne bénéficiera d'aucun avantage patrimonial avant sa propre mort, Artus rétorque : «Hector, ce dist Artus, nous sommes jones. Si en acquerrons asséz, et Diex nous aidera ${ }^{11}$. Quant aux demoiselles, elles ne sont pas en reste et affirment elles aussi leur statut de jeunes. Jehanette plaide ainsi la cause de celle qui est pourtant sa rivale, l'imprudente Péronne, et met en avant le fait que la jeunesse est «moult fort chose [...] a passer ${ }^{12}$. Pour autant, personnages masculins et personnages féminins ne connaissent pas un traitement semblable face à ce thème.

Le point de départ du roman est en effet la revendication continuelle de leur jeunesse par les personnages, revendication qui justifie la liberté qu'ils s'arrogent, au détriment des valeurs anciennes représentées par la génération des parents. Artus est étouffé par une mère possessive qui veut le garder auprès de lui : " mes la mere ne pooit sousfrir qu'il s'esloignast de li I seul jour, tant l'amoit chierement $»^{13}$. Il est également promis à une femme qu'il n'aime pas et décide de partir en « estranges païs ${ }^{14}$. Ce projet de départ est motivé par le souci de donner du lustre à sa jeunesse, comme le souligne Gouvernal par un proverbial : «jones homs sanz paine ne valt riens » ${ }^{15}$. Leur départ en aventures est ainsi placé sous le signe de la jeunesse et de ses oripeaux : " et furent lui et Hector et Gouvernaus en chauces d'escarlate, chascun I chapelet en sa teste comme josne homme ${ }^{16}$. De cette attitude, le héros tire une foi en l'avenir et un optimisme sans limite, notamment lorsqu'il assure à Hector qu'ils gagneront sans peine terres et biens ${ }^{17}$.

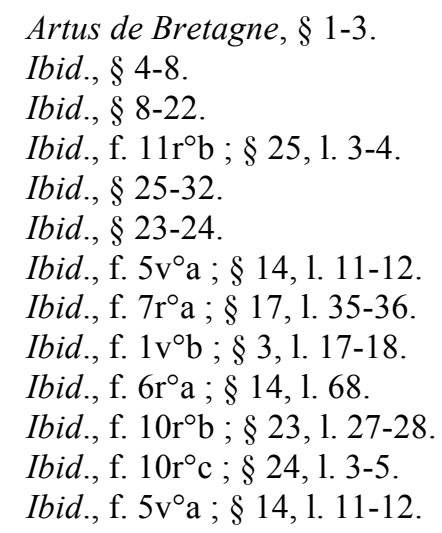


Au-delà d'une simple revendication de valeurs nouvelles contre l'ancienne génération représentée par les parents, l'insistance que mettent les personnages à faire de leur jeunesse la principale motivation à leurs actions est également l'occasion pour le romancier de mettre à distance le monde littéraire ancien. Ainsi Artus, comme Perceval dans le Conte du Graal, subit l'influence d'une mère abusive, castratrice et étouffante ${ }^{18}$. Mais contrairement au personnage de nice créé par CHRÉTIEN DE TROYES, Artus s'affranchit de l'influence maternelle avec autorité et en toute lucidité : il répond ainsi à sa mère, qui veut lui donner pour épouse une femme sur laquelle pèse le soupçon insistant du déshonneur : «Me tenéz vous si poi chier que vous me vouléz donner a personne diffamee ? ${ }^{19}$. Franche et nette, la parole d'Artus à ses parents est une première prise de distance avec ceux-ci. Il est conscient de ce qu'il est et des pressions qui s'exercent sur lui, pressions dont il se dégage par une parole qui affirme immédiatement sa liberté. Dès lors, sa quête ne sera pas une quête de soi, pas plus qu'elle ne sera une quête de l'amour ou de Dieu. De ce point de vue, l'Artus de Bretagne s'affranchit des modèles de romans anciens: Artus est un héritier amoureux dès le début du roman et il le reste jusqu'au moment de devenir un seigneur marié, même si in fine, il épouse une autre femme que Jeannette et se retrouve à la tête d'une autre terre que celle de son père. Au risque de paraître traité avec désinvolture, le motif du départ en aventure n'est motivé que par l'enthousiasme et la fougue de la jeunesse. Il n'y a aucune révélation à la clef du roman. Pour le héros, comme pour ses compagnons, la jeunesse est un tremplin vers le vaste monde qui s'ouvre alors à eux, sans réelle difficulté. Elle est ce qui permet de sortir de ce début de roman balbutiant et répétitif, enfermé par la reprise de schémas narratifs anciens, et fait entrer dans un récit neuf dont les codes vont peu à peu se définir.

De leur côté, les personnages féminins savent également se dresser face au conservatisme de leur parent lorsqu'elles estiment que cela est nécessaire. Jehanette, «fillete jone de l'eage de XIIII ans $\gg{ }^{20}$, prend ainsi la défense de Péronne qui s'est livrée aux plaisirs de la chair avant les noces et prône une sexualité qui s'assume librement en dehors du mariage :

« Madame, dist Jehanete, c'est moult fort chose que josnesche a passer et de destorber a femme ce qu'ele a entrepris. Sa fille, qui a esté a aise et souef nourrie, a qui chascuns estoit desirrans de lui servir a gré, a mespris. Pour Dieu, madame, regardéz amour qui l'a sozprise, quar ele amoit et estoit amee, et de l'autre part aaise la ou ele fu nourrie et jonesce a trespasser. Et qui jone fu onques ou qui l'est, ait pitié de la fille ou jonesce est assise en norreture et en amour de cuer » (f. $7 \mathrm{r}^{\circ} \mathrm{a}-7 \mathrm{r}^{\circ} \mathrm{b} ; \S 17,1.35-43$ ).

Loin de plaider l'erreur de jeunesse, Jehanette revendique l'amour et le plaisir sexuel comme un apanage des jeunes gens : ils sont une conséquence normale de la passion amoureuse qui, en toute bonne logique ovidienne, «l'a sozprise $»^{21}$, justement parce qu'elle a l'âge requis. À cet argument quasi médical, en tout cas physiologique et humoral, elle en ajoute un autre, social. Péronne n'a fait que jouer au jeu de la fin'amor auquel jouent depuis deux siècles toutes les héroïnes romanesques. En outre, la jeune fille réclame pour elle-même une part de cette liberté au nom de laquelle elle n'hésite pas non plus, à l'instar de Brangien, à prendre la place de Péronne dans le lit d'Arthur.

Mais la comparaison s'arrête là : quand les jeunes gens sont capables de briser ces carcans anciens pour échapper au poids des valeurs traditionnelles et impulser un nouveau souffle au roman, les jeunes filles s'avèrent prisonnières de ces codes anciens, en dépit de leurs tentatives pour les remettre en cause. Elles sont donc expulsées du récit : le roman fait mourir Péronne et abandonne Jehanette. Avec elles, d'autres univers littéraires sont mis à distance. Le mariage contraint avec Péronne, dissimulatrice aux mœurs légères, est orchestré par une dame de Luques peinte en maquerelle de fabliau. Les amours lacustres d'Artus et Jehanette ont été justement rapprochés par

18 Sur ce point on se reportera aux suggestions d'E. BAUMGARTnER (Chrétien de Troyes, Le Conte du Graal, Paris, PUF, « Études littéraires 62 », 1999) et de Fr. Dubost (Le Conte du Graal ou l'art de faire signe, Paris, Champion, «Unichamp 71», 1998).

19 Artus de Bretagne, f. $4 \mathrm{v}^{\circ} \mathrm{a} ; \S 11,1.17-18$.

20 Ibid., f. $2 \mathrm{r}^{\circ} \mathrm{a} ; \S 4,1.23$.

21 Pour une analyse de la mesure dans la peinture de l'amour, voir Ch. FERLAMPIN-ACHER, « Le charme d'Artus de Bretagne », Miscellanea mediaevalia, Mélanges offerts à Philippe Ménard, Paris, Champion, t. 1, 1998, p. 513-528. 
Christine FERLAMPIN-ACHER des schémas des lais féeriques ${ }^{22}$. Le personnage de Jehanette agrège d'ailleurs sur lui une foule de références littéraires : elle rappelle la Brangien de Tristan, la pastoure de la pastourelle, mais aussi certaines demoiselles libertines et dévergondées et prêtes à rompre serments et idéal courtois pour coucher avec Gauvain ${ }^{23}$. Péronne et Jeannette incarnent un modèle de jeunesse, certes, mais un modèle périmé et rejeté, un système de valeurs romanesques qui n'est pas celui que veut instaurer le romancier.

À l'ouverture du roman, la liberté des jeunes vis-à-vis des désirs ou des valeurs de leurs parents est donc un thème majeur mis en évidence par de très nombreuses références faites aux œuvres d'un passé littéraire avec lequel Artus de Bretagne entend prendre ses distances. Plusieurs portes ont été entr'ouvertes sur des possibles romanesques, toutes immédiatement refermées, condamnant certains personnages à l'oubli ou à la mort. Le seul passage possible est celui qui attire Artus et ses compagnons de voyage, avec toute la fougue de leur jeunesse, vers un ailleurs oriental que l'on espère neuf. Le personnage d'Artus tourne ainsi le dos à cet univers littéraire ancien, et la fonction de la première section du texte est de signifier que le roman part en quête d'une nouvelle jeunesse.

\section{Recyclage et neutralisation de l'héritage littéraire : aspects textuels}

Pourtant la matière qu'exploite l'Artus de Bretagne est loin d'être neuve, et l'on y retrouve une foule de motifs familiers de la littérature romanesque des XII ${ }^{\mathrm{e}}$ et XIII ${ }^{\mathrm{e}}$ siècles. Mais la façon dont ces motifs sont recyclés ne laisse pas d'être déconcertante car le roman s'applique à en neutraliser le fonctionnement avec une remarquable constance.

\section{Des motifs pour rire}

L'aventure merveilleuse offre un bon exemple de cette neutralisation : le roman fait en effet, à plusieurs reprises, écho à des motifs connus, puisés aussi bien dans la matière romanesque que dans la matière épique. Schématiquement, la merveille fait l'objet de deux types de traitement dans la littérature antérieure à Artus : soit elle possède une fonction de qualification du héros (type lit de la merveille dans le Conte du Graal ou déguisement de Guillaume à l'aide d'une herbe magique dans la Prise d'Orange), soit elle fait l'objet d'un traitement parodique qui la déconstruit (type lit de la merveille dans La Mule sans frein ${ }^{24}$. Or Christine FERLAMPIN-ACHER montre de façon convaincante que dans Artus l'aventure merveilleuse n'a pas de fonction qualifiante : "Artus est né héros et ne le devient pas $^{25}$. » De fait, l'épreuve du lit de la merveille ${ }^{26}$ ne sanctionne aucune compétence particulière du jeune homme : on savait depuis le songe $d u f .10 v^{\circ} b-10 v^{\circ} c(\S 24)$ et l'interprétation immédiate qu'en donne Gouvernal qu'Artus a un destin hors du commun, impliquant un mariage royal et le don d'une épée singulière. Les premières aventures que vit le

22 FERLAMPIN-ACHER Ch., «Artus de Bretagne : une histoire sans fin », art.cit., p. 56-57 et « D’un monde à l'autre : Artus de Bretagne entre mythe et littérature, de l'antiquaire à la fabrique de faux meubles bretons ", Le Monde et l'Autre Monde, D. HÜE et Ch. FERLAMPIN-ACHER (dir.), Actes du Colloque arthurien de Rennes (8-9 mars 2001), Orléans, Paradigme, 2002, p. 131-132.

23 On peut notamment citer la très libre demoiselle, fille du héros éponyme du Chevalier à l'épée (Two old French Gauvain Romances. Le Chevalier à l'épée and La Mule sans frein, éd. R.C. JOHNSTON et D.D.R. OWEN, Edinburg, R. \& R. Clark, 1972), et chez RaOul De Houdenc, l'Y dain de La Vengeance Raguidel (RaOul DE HoudenC, La Vengeance Raguidel, éd. G. RoussineAu, Genève, Droz, 2004).

24 Sur ces thèmes on consultera les études désormais classiques de D. POIRION (Le Merveilleux dans la littérature française du Moyen Âge, Paris, PUF, « Que sais-je », 1938, 1982), de M.-L. CHÊNERIE (Le Chevalier errant dans les romans arthuriens en vers des XII et XIII siècles, Genève, Droz, 1986), de Fr. DuBOST (Aspects fantastiques de la littérature narrative médiévale (XII ${ }^{e}$ XIII siècles). L'Autre, l'Ailleurs, l'Autrefois, Paris, Champion, 1991) et de J.R. Valette (La Poétique du merveilleux dans le Lancelot en prose, Paris, Champion, 1998), ainsi que celle d'I. ARseneAu (Parodie et Merveilleux dans le roman dit réaliste au XII siècle, Paris, Garnier, 2012). On se reportera également à l'article de Ch. FERLAMPIN-ACHER, « D'un monde à l'autre... », art.cit.

25 FERLAMPIN-ACHER Ch., «Épreuves, pièges et plaies dans Artus de Bretagne : le sourire du clerc et la violence du chevalier », La Violence dans le monde médiéval, Senefiance 36, Aix-en-Provence, 1994, p. 205.

26 Artus de Bretagne, § 92-94. 
jeune chevalier (aventure de la famille de la femme aux tresses coupées, le tournoi de Vienne ou bien l'épisode de Brueil) ne sont en rien qualifiantes, il n'y gagne rien, ni armes, ni terre, ni belle jeune fille. Son statut de jeune chevalier, héritier potentiel des terres paternelles, n'évolue pas. Elles ne sont que le moyen de confirmer ce que le songe a affirmé.

De surcroît, cette neutralisation de la fonction qualifiante de la merveille s'accompagne d'une description de l'épreuve du lit qui recycle jusqu'à la saturation les traits définitoires du motif que l'on trouve dans la littérature qui la précède ${ }^{27}$. Tout, dans les quatre paragraphes consacrés au lit de la merveille au château de la Porte Noire $^{28}$, relève de la surenchère hyperbolique par rapport au Conte du Graal: ce n'est pas un mais deux lions qu'Artus affronte ; le vilain armé d'un pieu qui, dans le roman de Chrétien de Troyes, faisait entrer le fauve dans la chambre, se mue en un géant à l'obstination féroce qui attaque le héros; le bruit de tonnerre qui retentit dans le palais des dames est remplacé par une voix prophétisant la fin des choses, tandis que se lèvent

si grant friente de gent et si grant noise qu'il sambloit bien qu'il y eust mil homes et ne veoit nului
fors que tant qu'il veoit chierges alumer par leens et estaindre plus de C. Si ooit aler et venir entour
le lit trop souvent et une riviere bruire, que vous deissiéz c'est la mer. Si en issoit uns vens si grans
que a grant paines pooit Artus estre sus ses piéz. (f. $36 \mathrm{r}^{\circ} \mathrm{c} ; \S 92,1.17-23$ )

Tout est à l'envi : la décoration du lit et de la chambre, les épreuves que doit surmonter le héros et les blessures qu'il reçoit. La merveille ainsi placée sous le signe de l'hyperbole parait d'autant plus massive et exagérée qu'elle n'en reste pas moins inopérante.

Le recyclage du merveilleux épique est lui aussi condamné à perdre sa fonction traditionnelle. Ainsi l'épisode d'Artus à la Clere Tour ${ }^{29}$ est l'occasion d'une récriture de la Prise d'Orange : tout comme Guillaume éprouve un manque douloureux dans Nîmes conquise, Artus se "melencolie » sur la route de la Clere Tour $^{30}$; tout comme Guillaume décide de s'introduire dans Orange la païenne avec deux compagnons, le visage noirci par la vertu d'une herbe, Artus s'entoure de cinq frères d'armes qu'Estienne grime en Sarrasins à l'aide d'un onguent magique pour pénétrer dans la Clere Tour tenue par les Babyloniens. Artus serait-il le nouveau Guillaume?

Las! Le passage montre que la vieille littérature épique ne trouve pas davantage grâce aux yeux de notre romancier que la tradition romanesque : la merveille et l'aventure n'y sont pas plus qualifiantes ici que là. Guillaume partait à la conquête d'une femme, d'une ville, d'une cour et d'un nom. Artus, lui, ne fait que traverser les lignes ennemies pour gagner une ville et une femme qui lui sont déjà acquises. Dans une perspective purement narrative, le merveilleux onguent ne sert de rien... L' « arrement [...] et autres herbes ${ }^{31}$ dont s'oignent Guillaume et ses hommes n'a, de fait, pas seulement pour effet de déguiser l'identité des Francs en travestissant leur apparence. Pour un temps, Guillaume est devenu un Sarrasin, accueilli comme tel par Aragon et sa cour, pourvu d'un passé et d'une mission que lui aurait confiée Thiebaut l'époux d'Orable, puis il est présenté à la reine. Pour un temps, il est contraint d'écouter les menaces que les païens profèrent à son encontre et de les approuver. Lui-même et un autre à la fois, Guillaume se regarde à travers les yeux de l'étranger. Mais ce dédoublement n'est pas faille chez le héros épique. Il est, au contraire, une étape sur le chemin qui le conduit à lui-même : pour que Guillaume de Narbonne conquérant de Nîmes puisse devenir à jamais Guillaume d'Orange, il lui faut endosser - pour la surpasser - la paienie d'Orange ; il lui faut conquérir Orable pour en faire Guibourc. Si le déguisement d'Artus et de ses compagnons, œuvre d'un maistre enchanteur, tient davantage que celui de Guillaume de la magie, il n'en a pourtant pas la puissance métamorphosante. Artus au visage noirci n'est que cela, un homme grimé, dissimulé sous une couche d'un parfait maquillage. Certes, cela lui permet de traverser les lignes ennemies et de pénétrer dans la Clere Tour pour rejoindre Florence. Cependant il ne s'agit pas de maîtriser les forces obscures qui l'habitent pour renaître plus grand, mais de

${ }^{27}$ Pour une étude du motif du lit de la merveille dans Artus de Bretagne et la littérature antérieure, envisagé sous l'aspect de sa valeur mythique, voir Ch. FERLAMPIN-ACHER, « D'un monde à l'autre... », art. cit., p. 136-152.

Ibid., § 440-453.

Ibid., f. $123 \mathrm{v}^{\circ} \mathrm{c} ; \S 440,1.8$, voir aussi f. $124 \mathrm{r}^{\circ} \mathrm{c} ; \S 442,1.32-33$.

31 La Prise d'Orange, éd. Cl. RÉGNIER, Paris, Klincksieck, 1986, v. 376-377. 
traverser avec le moins de heurts possible les lignes ennemies et de jouer une farce à la femme qu'il aime et dont il est aimé. Artus joue, de son corps et de sa parole, pour éprouver les sentiments de sa bien-aimée sans que jamais le moindre doute ne se fasse jour, sans que la moindre faille ne s'entr'ouvre entre les amoureux : Florence passe le test haut la main, certifiant son amour et sa fidélité. Il n'y a plus qu'à se démaquiller, en riant, pour qu'à nouveau l'être et le paraître coïncident.

«Sire, dist Artus a maistre Estene, ostéz moi ceste noirté que vous m'avéz donnee. » Lors rist li maistres moult fort et tuit li autre. [...]

Lors prist li maistres ses boistes. Si les oint et revindrent en leur premiere couleur. (f. $127 \mathrm{r}^{\circ} \mathrm{b}$; $\S 452,1.31$ à $\S 453,1.2)$

L'épisode ne revendique aucune valeur initiatique, il s'affirme comme un moment de divertissement et comme un jeu.

L'aventure merveilleuse, qu'elle trouve sa source dans le roman ou dans la chanson de geste, est donc soumise à un phénomène d'érosion qui la conduit à perdre son pouvoir qualifiant, alors que, dans le même temps, le récit qui en est fait s'alourdit sous le poids d'une surcharge intertextuelle hyperbolique. Ces deux caractéristiques, qui impliquent une mise à distance et un jeu avec un hypotexte reconnu, pourraient constituer les ingrédients d'un traitement parodique du motif. Or il n'en est rien : on ne trouve pas dans ces épisodes de regard distancié du narrateur ou d'indices textuels qui nous orienteraient vers une interprétation parodique du passage. De même, certains détails glissés au gré de descriptions de combats pourraient être burlesques. Mais ils ne fonctionnent pas sur un mode comique du fait qu'ils sont isolés dans des portions de texte où la tonalité sérieuse étouffe la potentialité burlesque. Ainsi, à son arrivée à la Porte Noire, Artus doit affronter pour les tuer les uns après les autres six chevaliers gardiens du pont-levis. Le deuxième connaît un sort particulier :

Si passa tout outre si roidement qu'il encontra si le secont qu'il jeta lui et le cheval sus le pont, et au relever qu'il quida faire, Bauduin le prist par le pié et le sacha a lui de dessus le pont en l'iaue. Si fu tantost noiéz. Si prist lors Bauduin la lance de celui et le bailla a Artus. Et si tost comme Artus la tint, si point et encontra un chevalier si roidement qu'il li fist passer parmi le cors, si qu'il l'abati mort. (f. $35 \mathrm{r}^{\circ} \mathrm{c} ; \S 88,1.9-17$ )

Ce chevalier, abattu, traîné par le pied puis jeté par dessus un pont, pourrait être le centre d'un tableau burlesque dans un épisode parodique si justement la trame textuelle de la parodie était plus resserrée. Or elle est très lâche et le comique ne prend pas ${ }^{32}$. Nous sommes au mieux face à des traits allusifs qui suscitent le sourire par l'évocation d'une tradition antérieure.

Dès lors, à quoi servent les aventures merveilleuses si elles ne sont pas moteurs de l'action ? À la fin de l'épisode de la Clere Tour, la noirceur du visage, métaphore de la bile noire qui envahit le héros, s'efface d'un simple geste du clerc qui emporte avec lui les troubles de l'âme. La légèreté de la jeunesse reprend alors le dessus. Ce qui semble compter, c'est le tourbillon d'aventures dont on sait qu'elles n'ont pas d'autre fonction que de célébrer la jeunesse du héros. Finalement, ce qui surprend dans l'Artus de Bretagne, c'est le contraste entre la complétude des motifs romanesques et la neutralisation de leur fonctionnement ancien qu'il soit potentiellement sérieux ou parodique. L'écriture par motifs trouve là une manière désinvolte de s'accomplir, une manière qui assume et exploite l'héritage littéraire mais le réduit à une forme d'insignifiance.

Pour mieux comprendre la fonction nouvelle de ces épisodes recyclés et allégés de leur fonctionnement ancien, il faut prendre en compte une autre dimension de la jeunesse telle qu'elle est représentée dans le roman : elle est inséparable d'une forme de rire nouveau, propre à ce roman.

Lorsqu'Artus, séparé de Florence, s'abîme dans la mélancolie, motivant ainsi l'épisode de la Clere Tour, ce n'est pas la première fois que le roman traite de ce motif. Au $\S 337$, alors que Florence feint la maladie pour retarder le mariage que veut lui imposer son père avec l'empereur

32 Contrairement, par exemple, à un épisode comme celui du combat de Gauvain contre un homme à la jambe de bois dans Hunbaut (Altfranzösischer Roman des XIII. Jahrhunderts, éd. W. FOERSTER, Dresden, Max Niemeyer, 1914). 
d'Inde, il est demandé à Estienne, censé la soigner, de « fai[re] fort le courecié » pour marquer son inquiétude ${ }^{33}$. Estienne s'exécute donc :

Si fu au piéz du lit, enbronchié en son chaperon, sa main a sa maissele. Si faisoit semblant d'estre trop courreciéz, si que Flourence et la royne en souzrioient tout couvertement et boutoient l'une l'autre. (f. $\left.101 \mathrm{v}^{\circ} \mathrm{c} ; \S 337,1.15-19\right)$

Le clerc prend la pose parfaite: le texte égrène les caractéristiques topiques du mélancolique ( «enbronchié », « main a [1]a maissele », « courreciez »). Toutefois il ne s'agit pas d'un portrait parodique : le rire des femmes nait au contraire du plaisir de voir une imitation parfaite, du bonheur de voir Estienne faire semblant, être à l'image de. Cette scène montre une imitation parfaite, pour le plus grand bonheur des femmes qui se délectent de la perfection de l'imitation en même temps qu'elles se réjouissent de savoir qu'il s'agit d'un jeu. Nous tenons là une des clefs du roman : le texte imite aussi bien qu'il le peut les modèles traditionnels et fait surgir un rire qui témoigne du plaisir éprouvé face à l'imitation parfaite d'un modèle connu, mais une imitation complètement décontextualisée et décalée, qui se met en spectacle. Ce rire est un rire communicatif, un de ces rires qui se transmettent par bourrade, un rire de connivence.

$\mathrm{Ce}$ rire inclusif et communicatif se retrouve tout au long du roman. Nous avons vu qu'Estienne efface la noirceur des visages grimés tout en riant et signe par ce geste le retour définitif des jeunes gens dans le cercle de Florence ( $\$$ 452-453). Auparavant, sur la route de la Clere Tour, Artus sort de ses réflexions mélancoliques grâce au rire que fait naître la beauté du chant d'Estienne :

Si commença a chanter en levant sa teste, en riant, et il chantoit a merveilles gracieusement si que li rois commença a rire et Phelippes et tuit li autre qui l'oürent. Et Artus s'esperist tout aussi comme s'il venist de pasmisons et lieve les ieux et vit le mestre et vit que li autre le regardoient. Si sousrist I petit par contenance. (f. $124 \mathrm{r}^{\circ} \mathrm{a} ; \S 441,1.3-8$ )

Le rire de ses compagnons contamine Artus qui est ainsi réintégré dans la troupe. Ce qui est célébré, c'est cette puissance du chant et de la voix dont la perfection crée un plaisir qui est décuplé d'être partagé.

Le plaisir de rejouer les scènes connues et de faire semblant est donc central dans Artus de Bretagne. C'est pourquoi l'humour épique hérité de la Prise d'Orange ${ }^{34}$ ou bien le sarcasme des femmes lors des joutes, tout droit venues du Tintagel du Conte du Graal ${ }^{35}$, sont massivement accompagnés de scènes de rire collectif où l'on voit les personnages réagir à l'instar de ce qui est attendu du lecteur, ce qui n'était pas le cas dans les textes anciens. Leur joie éclate ainsi à la vue d'un Artus déchaîné qui arrache des mains d'un serviteur le bras d'une charrette pour poursuivre le tournoi ( $\mathrm{Si}$ li esracha si roidement de la main qu'il l'envoia tout plat enmi le pre. Si encommencierent a rire les dames moult volentiers $\left.{ }^{36} »\right)$. Une pique bien sentie par laquelle la dame de Roussillon mouche la sœur du maréchal de Nevers, l'adversaire d'Artus lors du tournoi de Vienne, provoque également le rire des personnages («Lors commencierent les contesses a rire et tout torner a la bourde $\left.{ }^{37} »\right)$. Plus loin, ce sont Gouvernal et Hector qui rient d'Artus qui se retrouve à cheval sans selle à la suite d'un coup violent et ne s'est rendu compte de rien dans la fureur du combat (« et quant Artus se vit sanz sele, si s'en merveilla trop et demanda comment il estoit sanz sele et se sa sele li estoit cheoite. Si rist Hector et Gouvernaus asséz $\left.{ }^{38} »\right)$. D’autres éclats joyeux suivent et l'on rit du spectacle burlesque d'un chevalier combattant avec un tinel ou chevauchant à

33 Artus de Bretagne, f. $101 \mathrm{v}^{\circ} \mathrm{c} ; \S 337,1.14-15$.

34 Sur le sujet, voir J. FrapPIER, Les Chanson de geste du cycle de Guillaume d'Orange, t. II, Paris, Sedes, 1965, p. 255-317; Cl. LACHET, La Prise d'Orange ou la parodie courtoise d'une épopée, Paris, Champion, 1986, et La Prise d'Orange, Paris, Champion, CCMA, 2010, p. 63-72 ; D. BOUTET, « Le rire et le mélange des registres autour du cycle de Guillaume d'Orange », Plaisir de l'épopée, G. Mathieu-Castellani (dir.), Saint-Denis, PUV, 2000, p. 41-53.

35 Chrétien de Troyes, Le Conte du Graal, dans CEuvres, D. Poirion (dir.), Paris Gallimard, « La Pléiade », 1994, en particulier les v. 5060-5090.

36 Artus de Bretagne, f. $20 \mathrm{v}^{\circ} \mathrm{c} ; \S 43,1.40-42$.

37 Ibid., f. $22 \mathrm{r}^{\circ} \mathrm{b} ; \S 47,1$.97-98.

38 Ibid., f. $22 \mathrm{v}^{\circ} \mathrm{c} ; \S 48,1.75-78$. 
cru... Lorsqu'il éclate face à des scènes ou des procédés hérités des œuvres du passé, le rire des personnages ne peut pas être tout à fait un rire de découverte, spontané, c'est un rire de connivence qui inscrit dans le texte l'effet de rire au second degré que construit l'écriture romanesque.

Bien entendu, le risque de ce parti pris esthétique, c'est la lassitude que produisent la redite et les effets de citation massifs. Car le roman est essentiellement mu par une énergie citationnelle et non par une tension interne comme celle d'une quête guerrière ou amoureuse qui le pousserait vers une fin naturelle. Faute de posséder une réelle finalité, et pour ne pas s'enliser, le roman n'a pas d'autre choix que de mettre artificiellement un terme à cette longue revue littéraire qui le constitue. C'est pourquoi, voyant Artus incapable d'achever un combat lors d'un tournoi - et le roman impuissant à mettre un terme à sa propre matière - Estienne siffle la fin de partie. Alors un écran de fumée obscurcit brutalement la scène romanesque qui n'était finalement qu'un « faire semblant ${ }^{39}$ :

Si dist [Estienne] en son cuer : «Hé, gentilz chevalier, tu ne puez faillir aus tiens. Mainte hautes personnes sont, s'il fussent en vostre estat, que ja n'en fussent montéz a cheval, mes vous avéz cuer de gentil homme. Vraiement je ne poroie plus soufrir vostre travail ! " Lors sifla li maistres. Si fist venir au tornoy une si grant fumee que nulz ne vit l'autre. Si furent tuit esbahis dont ce venoit, et ainsi se departirent cil du paÿs. (f. $143 \mathrm{r}^{\circ} \mathrm{b} ; \S 467,1.95-103$ )

Explicit le roumans d'Artus le Restoré.

La fin du roman ne pouvait qu'être désinvolte elle aussi.

\section{Des visions pour rire}

Cependant, il n'y a pas que les motifs, comme l'aventure merveilleuse, le déguisement ou encore le rire, que l'Artus de Bretagne rajeunit en les réinvestissant d'une valeur nouvelle dans son entreprise de dépoussiérage de la matière littéraire ancienne. Cet effort touche également, à un niveau microstructural, l'écriture elle-même, comme en témoigne le traitement de la formule traditionnelle qui veïst ${ }^{40}$. L'intérêt de l'étude de cette formule dans notre roman réside dans la reconfiguration de son fonctionnement syntaxique et sémantique à laquelle le texte la soumet.

Volontiers qualifiée d'épique à cause de sa fréquence d'emploi dans la chanson de geste, cette formule permet de souligner une scène topique en impliquant le destinataire de la narration. Signe d'une oralité, fictive mais revendiquée, elle apparaît de ce fait plus volontiers dans les textes en vers et voit sa fréquence diminuer au fur et à mesure que le Moyen Âge avance. Les occurrences de notre roman en prose de la fin du XIII ${ }^{\mathrm{e}}$ siècle retiennent l'attention.

À deux reprises, Artus de Bretagne fait appel à la formule qui veïst. Une première fois pour montrer la réaction du clergé de la Roche face aux enchantements d'Estienne :

Qui lors veist moines, nonnains, clers et prestres courir aus eglises et confesser li uns l'autre, et

mettre aus genoulz et batre leur coupes de grant paour, li peust souvenir ! (f. $51 \mathrm{v}^{\circ} \mathrm{c} ; \S 133,1.1-4$ )

Une seconde fois pour souligner l'arrivée de Florence à Argens :

Qui donques veist les bourgois monter aus fenestres pour veoir Florence qui estoit montee sus I palefroi... (f. $\left.105 \mathrm{v}^{\circ} \mathrm{a}\right)^{41}$

Formellement, qui veïst est la protase d'un système hypothétique, et peut apparaître, avec ou sans son apodose. Dans tous les cas, la construction vise à établir un lien direct entre narrateur et lecteur, le récit du premier suscitant par la description de la protase des émotions de diverses natures chez le second (effroi, admiration, etc.). À première vue, les occurrences de notre roman représentent ces

39 FERLAMPIN-ACHER Ch., «Artus de Bretagne : une histoire sans fin », art. cit., p. 61-62.

40 Voir sur ce point N. BRAGANTINI-MAILLARD, «Retour sur le tour hypothétique qui veïst», Entre syntaxe et discours. Éclairages épistémologiques et descriptions linguistiques, Linx 62-63, Paris Ouest-Nanterre-La Défense, p. 181-196 (consulté en ligne le 28 mai 2014 : http://linx.revues.org/1384). Voir également Ph. MÉNARD, Syntaxe de l'Ancien Français, Bordeaux, Sobodi, 1973, § 76b rem. 1 ; G. Molgnet, Grammaire de l'Ancien Français, Paris, Klincksieck, 1988, p. 157 et 247; N. ANDRIEUX-REIX, "Lors veïssiez, histoire d'une marque de diction », Linguistique de l'énonciation - Approche diachronique, Linx 32, 1995, p. 133-145 ; Cl. BURIDANT, Grammaire nouvelle de l'ancien français, Paris, Sedes, 2000, § 536.

41 Preuve que le passage pose problème, l'éditrice choisit de corriger en «Et adonc veissiés les bourgois...» (\$ 355 , 1. 2-3), s'appuyant sur la leçon d'un autre manuscrit, et évite ainsi de présenter un système hypothétique tronqué. 
deux cas de figure (la première citation possède un système entier de protase et d'apodose : « li peust souvenir », la seconde étant sans apodose).

La construction sans apodose repose sur un implicite partagé entre narrateur et lecteur : la simple présence de la formule qui veïst dans la protase implique, de façon topique, une réaction émotionnelle dans l'apodose, réaction dont la nature est définie contextuellement. De fait, l'arrivée de l'héroïne Florence, son entrée solennelle, le peuple de la cité étant aux fenêtres, font supposer, sans qu'il soit besoin de le préciser, que le récit du narrateur et la vision introduite dans la protase sont connotés positivement. Point n'est besoin de l'expliciter par une apodose : la majesté de Florence et de son cortège est admirable.

Toutefois, la façon dont s'énonce la vision de la protase est étonnante. Que nous y est-il donné à voir? La foule émerveillée des bourgeois qui se presse pour voir l'entrée de Florence dans la ville d'Argençon... Ce n'est donc pas Florence que le public est invité à imaginer, mais bien les bourgeois admirant Florence. Un relai du regard est ainsi mis en place qui suggère une vision indirecte. En nous donnant à voir un personnage qui regarde un autre personnage, le texte attend-il finalement du lecteur qu'il s'émerveille de Florence ? Il semblerait plutôt que le lecteur soit invité à s'émerveiller d'un émerveillement plutôt que de ce qui le provoque.

C'est exactement le contraire de ce qui serait normalement attendu d'une formule qui fait appel à une forte connivence entre narrateur et lecteur et qui prétend abolir les écrans entre le récit, ses effets et le lecteur à qui ces effets sont destinés. L'émotion est ici relayée non pas par le narrateur mais par les personnages dont la vision par le lecteur doit permettre à ce dernier de prendre la mesure de l'admiration que suscite le tableau de l'arrivée de Florence. L'émotion ne se construit donc pas sur le plan du discours, mais sur le plan du récit, et l'effet subjectif que doit créer la formule sur le lecteur est neutralisé.

À regarder la première occurrence de qui veïst, il semblerait que celle-ci fonctionne elle aussi de façon particulière. L'apodose « li peust souvenir », qui signifie que le lecteur qui verrait la scène s'en souviendrait longtemps et vivement, utilise le verbe souvenir sans objet exprimé et en construction impersonnelle, comme c'est possible en ancien français. Toutefois, le choix d'un emploi non transitif direct de ce verbe soustrait de l'apodose un élément sémantiquement essentiel : l'objet du souvenir, son contenu, qui n'est autre que la vision de panique des nonnes et des prêtres décrite dans la protase. Sans complément d'objet à souvenir, le lien sémantique et référentiel entre protase et apodose est dissout, si bien que le verbe se retrouve dépourvu de support concret.

Or le rôle du second volet du système hypothétique est de développer le COD de veist et d'expliciter ce qu'il y a de si remarquable à voir pour que l'on sollicite la présence d'un publictémoin dont le regard est régi et façonné par la description de la protase. Cette apodose, qui gomme le contenu du souvenir, fonctionne donc de façon similaire au précédent exemple dans la mesure où ce que la vision doit susciter est soustrait de la phrase, si bien que la réaction subjective du lecteur est une fois encore neutralisée.

L'appel au souvenir est étrange : le fonctionnement mémoriel romanesque est finalement un leurre puisque l'appel à une mise en mémoire du récit est ainsi fait qu'il ne peut pas fonctionner. Voilà qui est paradoxal dans un roman de veine arthurienne, roman qui de surcroît s'ouvre sous l'égide de la « ramembrance ». En effet, Artus est ainsi prénommé en souvenir du roi de Logres :

Li dus Jehans ot I enfant de sa femme, qu'il firent apeler en la ramembrance de la haute renommee du bon roy Artu, et li donnerent non d'Artus. (f. $1 \mathrm{v}^{\circ} \mathrm{a} ; \S 3,1.1-3$ )

Mais c'est là l'unique occurrence du mot ramembrance, notion pourtant essentielle pour comprendre le sens et le fonctionnement de l'écriture romanesque médiévale arthurienne en tant que mise en mémoire du passé. Cette mise en avant du souvenir, dès ses premières lignes, puis l'effacement concerté de cette notion est un indice du rapport qu'entretient le roman à son héritage littéraire : si cet héritage est pleinement exploité au cours des aventures d'Artus, il est dépouillé de son sens et de sa fonction originels. De même, à un niveau microstructural, l'appel au souvenir se double d'une neutralisation de celui-ci, dans un mouvement paradoxal d'invocation et de révocation. 
Dans ces exemples, on l'aura compris, la connivence entre narrateur et narrataire ne se tisse pas grâce au fonctionnement topique de la formule qui veïst, mais à travers une sorte de plaisir du second degré. Venue du passé, puisée dans la tradition littéraire, elle est vidée de sa force originelle. Mais elle fait sens par sa simple présence, au même titre que les motifs détournés. Pour l'auteur d'Artus de Bretagne, renouveler la matière romanesque ne signifie pas faire table rase du passé, mais jouer avec lui.

Le jeu se poursuit d'ailleurs avec un écho à cette formule que l'on trouve au $\S 208$. Pendant les préparatifs pour le duel judiciaire qui doit décider du sort de Marguerite d'Argençon, après avoir embrassé les reliques et juré de faire éclater le droit de la demoiselle, Artus enfourche son destrier :

il deist qui le veist qu'il fust nez atout le cheval. (f. $69 \mathrm{v}^{\circ} \mathrm{a} ; \S 208,1.16-17$ )

Ce qui met notre intérêt en éveil dans cette citation, c'est l'écho qui fait résonne la formule qui la/lors veïst. Certes, nous sommes très simplement face à une phrase dont la complétive (« qu'il fust nez atout le cheval ») est le COD d'un verbe régissant (« deist») qui a pour sujet le pronom « il », développé par la relative appositive « qui le veist»: «il aurait pu dire, celui qui l'aurait vu, qu'il faisait corps avec sa monture ». Cependant la phrase fonctionne phoniquement comme un écho postiche ou pastiche à la formule qui la veïst. Dans « qui le veist », le pronom le tient la place d'un adverbe de lieu « la », comme si, par anticipation, une partie de l'apodose remontait en amont de la $\operatorname{protase}^{42}$. Il y a ici, une fois encore, un jeu concerté avec l'évocation d'une formule ancienne qui ne fonctionne pas et suscite une vision qui fait échouer la relation de connivence entre narrateur et lecteur. Certes, la vision de l'Artus centaure a bien lieu. Ce qui est donné à voir mérite le détour : l'eslais d'Artus manifeste l'excellence d'un chevalier digne, en tous points, d'admiration. Mais l'hésitation et le flou installés dans la lecture maintiennent une fois encore le lecteur et son émotion à une distance respectable. Si connivence avec le lecteur il y a, celle-ci ne passe pas par le discours d'un narrateur à l'ancienne mode, mais par le discours d'un narrateur qui fait imploser les cadres anciens qui fixaient sa relation au lecteur. Le geste est irrévérencieux, désinvolte, mais fonde une esthétique du second degré propre à notre roman.

La topique et la rhétorique du qui veïst sont donc bien détournées dans notre roman: réminiscence littéraire privée de l'épaisseur de sens dont la tradition littéraire l'avait dotée, la formule tantôt met l'accent sur le banal et l'insignifiant, tantôt se désintéresse de la mise en mémoire du récit. La vision est détournée de sa valeur exemplaire ou émotionnelle, et le lecteur n'est pas tenu de la garder en mémoire. Le plaisir qu'il en tire est celui de la reconnaissance nonnostalgique d'une trace du passé littéraire remise au goût du jour et allégée du poids de son fonctionnement ancien.

\section{Recyclage et neutralisation de l'héritage littéraire : aspects péritextuels et iconographiques}

Ce jeu avec la topique et la rhétorique apparaît comme une caractéristique de notre roman. Il est si marquant que l'un des deux codex qui nous a transmis cette version du texte l'inscrit dans la matérialité de l'objet-livre, à deux niveaux différents, celui de la rubrication et celui de l'enluminure.

\section{Rubriques à brac}

L'organisation visuelle et matérielle du texte du manuscrit de Paris (BnF français 761) porte la trace de la même désinvolture, que l'on peut considérer comme un principe poétique de création de ce roman, aussi bien dans sa facture textuelle que matérielle et iconographique. En effet, si la plupart des rubriques et les paragraphes remplissent leur fonction d'organisation du roman, d'autres

42 N. BRAgAntini-Maillard, dans « Retour sur le tour hypothétique qui veïst», art. cit., signale des possibilités d'anticipation dans la protase d'éléments de l'apodose (p. 182-183). 
nous paraissent avoir une utilité structurante plus discutable, si bien qu'il faut chercher ailleurs leur fonction.

Nous observons que certaines rubriques de ce codex délimitent et mettent en valeur des portions de texte surprenantes. Par exemple, la rubrique du $\S 334$ (« Comment Artus vint au tref le roy d'Orcanie », f. $\left.101 r^{\circ} a\right)$ annonce un événement absolument anodin qui se situe à la fin du court paragraphe qui suit, à savoir le trajet qu'effectue Artus entre la tente de Florence et celle du roi d'Orcanie. La rubrique, en soulignant un moment insignifiant de la diégèse, passe sous silence un événement qui est, du point de vue de l'efficace narrative, bien plus essentiel : la révélation faite au héros par Estienne du stratagème de Florence pour retarder la deuxième journée du tournoi.

Une autre séquence est particulièrement instructive : le $\S 52$ s'achève sur les deux phrases suivantes :

Lors furent la venues toutes les contesses et les autres dames, qui bien furent de cest acort qu'il fust coronnéz comme le meilleur chevalier de tous. Si fu aportee la couronne. (f. $24 \mathrm{v}^{\circ} \mathrm{b} ; \S 52$, 1. 37-40)

Puis le $\S 53$ est annoncé par la rubrique suivante : «Comment li roys de Maillogres couronna Artus comme le meilleur chevalier que l'en peust trover par le gré de tous les contes et de tous les barons qui la furent et des contesses aussi » (f. $24 \mathrm{v}^{\circ}$ b). Le très bref $\S 53$ se déploie ensuite sur deux phrases et quelques lignes :

Lors le couronna li roys de Maillogres de commun acort pour estre roys des chevaliers et li promistent secours et aide en tous lieux encontre tous et a tant de fois en tous les lieux que il voudroit. Si firent joie et feste moult grant qui dura bien XV jors tous plains. (f. $24 \mathrm{v}^{\circ} \mathrm{b} ; \S 53$ )

Seule la première proposition de la rubrique («Comment li roys de Maillogres couronna Artus ») annonce le contenu du paragraphe suivant, et ce de façon pléonastique puisque le $\S 52$ annonçait déjà le couronnement d'Artus. On pourra objecter que le rôle de la rubrique est de sortir du texte des éléments structurants qui sont mis visuellement en valeur. Il n'y aurait pas de ce point de vue de pléonasme. Toutefois cette rubrique brise l'avancée du récit et ne possède aucune fonction proleptique dans la mesure où elle est tautologique et ne fait finalement que reprendre des éléments déjà connus (couronnement, agrément de la cour). Par ailleurs, la brièveté du $\S 53$ empêche justement la rubrique de fonctionner de façon authentiquement proleptique : aucun durée narrative ne vient s'insérer entre la rubrique et ce qu'elle annonce. Le phénomène mémoriel qu'implique la prolepse ne peut se mettre en place. Le paragraphe n'est qu'une redite de la rubrique. L'organisation matérielle du texte brode sur ce que le lecteur sait déjà et entrave le bon fonctionnement de la rubrication que l'on préfère exhiber plutôt que de l'éliminer. Le manuscrit préfère une fois encore la présence de l'insignifiant à l'absence pure et simple de rubrique.

À quoi servent donc ces rubriques qui n'annoncent rien? Ne sont-elles qu'un ornement visuel, une tache de couleur dans une page d'écriture ? Un troisième exemple, au fonctionnement un peu différent, nous permet d'avancer une hypothèse. Au f. $53 \mathrm{r}^{\circ} \mathrm{c}$ nous trouvons la rubrique suivante :

Comment Flourence fu si esprise de l'amor Artus qu'ele en perdi la parole, et quant ele fu revenue, si dist que c'estoit de la paor de la teste du moustre, et li roys la fist tantost ardoir, et comment Artus tua I lyon, I jaiant, une jaiande et I grisfon, et monseigneur Rogier le cousin au neveu le duc de Bigorre. (f. $53 \mathrm{r}^{\circ} \mathrm{c} ; \mathrm{p} .187$ )

Cette rubrique possède une réelle utilité narrative puisqu'elle résume très bien la portion de texte qui s'étend des $\S 137$ à 155 . Elle n'a cependant pas qu'une fonction proleptique. Stylistiquement, elle est classiquement construite comme une interrogative indirecte dont la fonction est de résumer les événements qui vont être narrés. Pourtant, par une sorte d'effet de porosité fonctionnelle, cette rubrique se fait elle-même récit : elle s'enrichit de la parole des personnages en insérant en son sein un court dialogue rapporté au style indirect entre Florence et son père : "si dist que c'estoit de la paor de la teste du moustre, et li roys la fist tantost ardoir ». Elle concurrence même directement le narrateur principal en scandant de conjonctions et d'adverbes les étapes du récit dont elle adopte la logique narrative : « et quant ele fu revenue, si dist que c'estoit de la paor de la teste du moustre, et li roys la fist tantost ardoir $»$ (nous soulignons). Parallèlement à cette incursion hors de sa syntaxe 
habituelle, la rubrique du $\S 137$ exploite de façon comique la capacité d'énumération accumulative que permet l'écriture traditionnelle de la rubrique. Elle énumère les actions de Florence, de son père et d'Artus et, dans la proposition dévolue à Artus, elle égrène de façon rythmée quatre segments trisyllabiques, auxquels s'en ajoute un beaucoup plus long. L'ensemble crée un effet comique de cadence majeure et de clausule puisque le segment « monseigneur Rogier le cousin au neveu le duc de Bigorre " clôt par un contre-pied un inventaire à la Prévert de merveilles composée d'un lion, d'un géant et d'une géante et d'un griffon. L'impression de bric et de broc fait naître le sourire, mais l'essentiel de l'information est transmis, et le lecteur sait tout ce qu'il y a à savoir de la narration à venir. En brillant par sa forme attrayante et soignée, la rubrique fait une sérieuse concurrence au texte qu'elle annonce et qui n'est jamais plus qu'une amplification dont l'essentiel, en termes de contenu comme de forme, a déjà été dit.

En n'annonçant rien ou pas grand chose, la rubrique produit donc de l'insignifiance. Et en concurrençant le récit lui-même, elle en neutralise la valeur d'événement, le poussant à ne plus fonctionner que comme une redite, si bien que la forme visuelle du texte ne délimite plus des unités narratives pertinentes. L'organisation péritextuelle du roman investit et pervertit une forme-sens traditionnelle du manuscrit pour en neutraliser la fonction et la réduire à l'insignifiance.

L'examen de quelques miniatures confirme le constat selon lequel le codex, dans sa matérialité même, est touché par cette volonté de faire peau neuve.

\section{La désinvolture de main de Maître}

Deux manuscrits jumeaux nous ont transmis la version courte ${ }^{43}$ du texte : le BnF français 761 (54 miniatures réalisées par le MAÎTRE DE FAUVEL) et le codex 403 de la Bibliothèque Inguimbertine de Carpentras (37 miniatures de plusieurs mains). Les deux programmes iconographiques sont parents, mais pas identiques, et ils proposent des lectures différentes du $\operatorname{roman}^{44}$.

Ce qui frappe en premier lieu dans la série d'enluminures du MAÎTRE DE FAUVEL, c'est l'utilisation massive de patrons compositionnels qui imposent au fil du manuscrit la répétition d'un même schéma de tableau en tableau. Nous observons ainsi que, dans l'illustration des scènes de bataille ou de duel, Artus est représenté majoritairement sur la droite de l'image, monté sur un cheval dont le caparaçon est orné d'un lion rampant, le bras droit en extension frappant son ennemi. La représentation de Gouvernau et d'Hector utilise également cette formule visuelle figée qui est un trait caractéristique du style du MAÎTRE DE FAUVEL puisque nous la retrouvons par exemple dans son illustration de l'Historia de GUILlaUME DE TYR ou du Merlin ${ }^{45}$. Cette formule iconographique neutralise le lien entre texte et image sur le plan narratif mais aussi générique : sa répétition, sans inscription de détails propres aux événements contés et susceptible de les rattacher à un épisode ou à une matière précise, déstructure le lien qui unit le cycle iconographique au récit romanesque et ce de manière massive puisque dans Artus, c'est un total d'environ $40 \%$ des images qui est concerné.

43 Woledge B., « Les manuscrits du Petit Artus de Bretagne », Romania 63, 1937, p. 393-397 ; SPILSBURY S., « Artus de Bretagne: structure and unity », Romania, 97, 1976, p. 63-67 ; FERLAMPIN-ACHER Ch., "Artus de Bretagne: une histoire sans fin », art.cit., p. 53-68. Nous renvoyons, pour la consultation du manuscrit de Paris à Gallica pour le manuscrit de Paris (http://gallica.bnf.fr/ark:/12148/btv1b84478715/f1.image, page consultée le 28 mai 2014), à Mandragore (mandragore.bnf.fr) pour ses illustrations, et pour le manuscrit de Carpentras à la Bibliothèque Virtuelle des Manuscrits

Médiévaux (http://bvmm.irht.cnrs.fr/resultRecherche/resultRecherche.php?COMPOSITION_ID=10536, page consultée le 28 mai 2014).

44 Pour une approche des programmes iconographiques des différents manuscrits d'Artus de Bretagne, on peut se reporter à l'étude de Ch. FERLAMPIN-ACHER, «Grandeur et décadence du clerc Estienne dans Artus de Bretagne », Le Clerc au Moyen Âge, Senefiance 37, Aix-en-Provence, 1995, p. 182-195.

45 Voir Artus, BnF français 761 (f. 9r $\mathrm{r}^{\circ}, 19 \mathrm{v}^{\circ}, 27 \mathrm{r}^{\circ}, 30 \mathrm{r}^{\circ}, 45 \mathrm{v}^{\circ}, 47 \mathrm{v}^{\circ}, 108 \mathrm{v}^{\circ}, 112 \mathrm{v}^{\circ}, 143 \mathrm{r}^{\circ}$ ), GUILLAUME DE TYR, Historia, BnF français 22495 (f. $79 \mathrm{v}^{\circ}, 95 \mathrm{r}^{\circ}, 105 \mathrm{v}^{\circ}, 106 \mathrm{v}^{\circ}, 109^{\circ} \mathrm{v}, 27^{\circ} 1 \mathrm{v}$ ) (consultable sur mandragore.bnf.fr et sur http://gallica.bnf.fr/ark:/12148/btv1b9062228b/f2.image, page vue le 28 mai 2014), Histoire de Merlin, BnF français 105 (f. $116 \mathrm{v}^{\circ}, 133 \mathrm{v}^{\circ}, 146 \mathrm{v}^{\circ}, 187 \mathrm{r}^{\circ}, 194 \mathrm{v}^{\circ}, 205 \mathrm{r}^{\circ}, 222 \mathrm{v}^{\circ}, 234 \mathrm{r}^{\circ}, 237 \mathrm{v}^{\circ}, 239 \mathrm{r}^{\circ}$ ) (consultable sur mandragore.bnf.fr et sur Gallica http://gallica.bnf.fr/ark:/12148/btv1b90591425.r=Merlin.langFR, page vue le 28 mai 2014). 
Par comparaison, les enlumineurs du cycle iconographique de Carpentras ont tenté de resserrer le lien entre texte et image en réduisant le nombre de scènes de combat $(11-$ dont 2 présumées car le manuscrit de Carpentras est lacunaire - contre 19 dans le manuscrit de Paris) et en les singularisant. Par exemple, si dans ce manuscrit les scènes de bataille dissimulent les chevaliers derrière leur heaume, la dernière miniature montre les visages de tous les combattants à l'exception d'un seul. Cette image prend en compte la rubrique : «Comment Artus vainqui le tournoy lui et Gouvernaus mais nulz ne les congnoissoit pas pour leurs armes » (f. $\left.163 \mathrm{r}^{\circ}\right)$. Le jeu de caché-montré des visages reformule visuellement le propos du texte sur l'anonymat des deux compagnons lors du tournoi. De même, au f. $151 \mathrm{r}^{\circ}$, l'image illustre le cycle de vengeances explicité dans la rubrique : «Comment Floripas [...] occist le roi de Morival [...] et comment Artus tua le roy Floripas ». L'image représente le combat qui oppose le roi Morival à Floripas, le roi Morival mort, mais aussi le coup porté par Artus à Floripas, dans une concaténation des temporalités propre à l'iconographie médiévale. Nous n'observons en revanche rien de tout cela dans le manuscrit de Paris (f. $133 \mathrm{v}^{\circ}$ ) qui se contente d'une simple scène de bataille rangée dans laquelle Artus tue Floripas : l'enchaînement des événements est absent et l'image tend davantage vers le cliché iconographique, le topos de la mêlée, que vers la représentation d'un épisode précis.

Cette illustration par stéréotype, caractéristique du manuscrit de Paris, serait-elle tout simplement un goût, une marque de fabrique de l'enluminure médiévale en général et du MAîTRE DE FAUVEL en particulier ? Dans l'image où Artus se grime en Sarrasin (f. $123 v^{\circ}$ ), rien n'illustre le déguisement : l'on ne voit, banalement, que des chevaliers se rendant à un château. Une fois encore l'image évite la singularité de la narration pour se réfugier dans une représentation banale. Pourtant ni la technique, ni le savoir-faire du MAÎTRE DE FAUVEL ne sont à mettre en cause comme en témoigne la façon dont il a rendu la peau noire des Sarrasins dans son manuscrit de l'Historia de Guillaume DE Tyr ou celle des Saxons dans 1'Histoire de Merlin ${ }^{46}$. Dans Artus, le cycle iconographique du MAÎTRE DE FAUVEL tend intentionnellement à ne pas singulariser les images à partir des faits de la diégèse, alors que dans le manuscrit de Carpentras (f. 139r ${ }^{\circ}$ ), l'image tente clairement d'adapter visuellement le contenu du texte, et l'on voit Artus et ses compagnons la peau noircie, s'approchant d'un château clairement identifiable comme étant celui mentionnée par le texte grâce aux dames à ses fenêtres et aux Sarrasins aux créneaux ${ }^{47}$.

Un autre exemple illustre cette tendance du BnF français 761 au stéréotype et celle des peintres du codex carpentrassien à la refuser : quand le MAÎTRE DE FAUVEL propose une scène banale (f. $\left.66 \mathrm{v}^{\circ}\right)$ montrant un groupe arrivant en un lieu quelconque, dont on ne saurait dire, sans son cotexte, l'épisode précis qu'elle illustre (et de ce point de vue elle fonctionne de la même façon que notre exemple de la rubrique du $\S 334$ commenté plus haut ou de la même façon que l'image du f. $133 \mathrm{v}^{\circ}$ ), le manuscrit de Carpentras (f. $78 \mathrm{r}^{\circ}$ ) représente Estienne dans la posture du maître donnant une leçon sur le cosmos et dirigeant le regard au moyen de son index vers un ciel rempli d'étoiles, conformément au texte qui accompagne les deux témoins de la version courte ${ }^{48}$.

Sous forme de pied de nez, et comme pour souligner que l'illustration s'attache en priorité à des événements secondaires, l'image qui suit, dans le BnF français 761, celle où nous voyons Artus entrer dans la Clere Tour, montre Artus sortant de la Porte Noire. Les deux enluminures (f. $123 \mathrm{v}^{\circ}$ et f. $127 \mathrm{v}^{\circ}$ ) sont construites en symétrie inverse: des chevaliers arrivant dans un château, des

46 Guillaume De TYR, Historia, BnF français 22495 (f. $9 \mathrm{r}^{\circ}, 16 \mathrm{v}^{\circ}, 28 \mathrm{r}^{\circ}, 30 \mathrm{r}^{\circ}, 32 \mathrm{v}^{\circ}$, etc.) et Histoire de Merlin, BnF français 105 (f. $216 \mathrm{r}^{\circ}, 218 \mathrm{r}^{\circ}, 220 \mathrm{r}^{\circ}, 223 \mathrm{v}^{\circ}$, etc.).

47 Le texte dit des Sarrasins : " $\mathrm{Si}$ en vint plus de $\mathrm{V}^{\mathrm{C}}$ a la porte et hurterent, et tantost la guete cria aus armes quanques il pot. Lors saillirent tuit cil du chastel aus fenestres »(f. $\left.125 \mathrm{v}^{\circ} ; \S 449,1.11-14\right)$. Les dames aux fenêtres représentent Florence et ses dames détenues à la Tour Clere.

48 Les rubriques des deux manuscrits mentionnent toutes les deux très clairement la leçon d'astronomie : « Comment maistres Estene devise a Marguerite, la dame d'Argençon, la signification de la terre, de la lune, du solel et des estoiles et de tout le firmament, et comment il vindrent a la Porte Noire a son oncle, la ou il firent trop grant joie » (f. $66 v^{\circ}$, p. 256) et « Comment mestre Estienne devisse a Marguerite d'Argançon la significacion de la terre et de la lune, du souleil et des estoilles. Et comment il vindrent à a la Pore (sic) Noire a son oncle, la ou il firent moult grant joie » (Bibliothèque Inguimbertine 403, f. $78 \mathrm{r}^{\circ}$ ). 
chevaliers partant d'un château. Seule la lecture du texte permet de savoir que la narration a progressé entre ces deux instants et que les châteaux sont deux lieux différents. Ce qui compte pour les images, ce sont les mouvements, qui encadrent des épisodes non illustrés ; il n'y a pas d'intérêt pour les événements romanesques ou épiques. Le cycle iconographique insiste ainsi sur ses omissions volontaires, sur son refus de scènes que l'on se serait attendu à voir comme les combats contre les Sarrasins ou la rencontre avec Florence. Au lieu de montrer une scène attendue dont elle enrichirait le sens, l'image déjoue ce sens et le fait sombrer dans une forme de banalité déceptive. L'image crée de l'insignifiant à la façon de certains paragraphes et de certaines rubriques que nous relevions plus haut : la matière romanesque et la matière épique traditionnelles y sont dissoutes. Les enlumineurs du manuscrit de Carpentras corrigent cet effet déceptif et témoignent de l'horizon d'attente qui pouvait être celui d'un lecteur médiéval en terme d'illustration du roman, et donnent notamment une idée du degré minimal de narrativité et de référentialité attendu, degré que n'atteint pas le manuscrit de Paris. L'illustration d'Artus dans sa version première ${ }^{49}$, celle du MAÎTRE DE FAUVEL, relève donc d'une forme d'esthétique intentionnelle de la désinvolture qui déjoue les attentes des lecteurs.

Tout l'intérêt de ces remarques est de faire observer que le MAÎTRE DE FAUVEL relaie dans son programme iconographique l'esthétique textuelle de la désinvolture que nous avons mise au jour. Une comparaison de certains traits poétiques du roman avec la fabrique des images est révélatrice de la forte cohérence esthétique du manuscrit de Paris et de l'intelligence de son enlumineur qui se cache derrière son apparente banalité. En effet, ses images peuvent être rapprochées des formules syntaxiques du type qui la veïst. De la même façon que le romancier les vide de leur fonction première de régie et d'encadrement du regard pour reconstruire un regard nouveau, les enluminures du BnF français 761 détournent la fonction délimitante des cadres de l'image pour orienter le regard $\mathrm{du}$ lecteur. Nous notons en effet un élément curieux : les encadrements ne sont pas peints de façon homogène. Là où l'on attendrait des bords bleus ou rouges - pratique très courante dans l'enluminure contemporaine du MAÎTRE DE FAUVEL - ils sont le plus souvent bleus et rouges. Nous pouvons avancer l'hypothèse que ce mélange chromatique a pour fonction de souligner les éléments importants de l'image en plus de/ou plutôt que d'en offrir une simple découpe. Sans qu'ils soient chargés d'un sémantisme particulier, le bleu et le rouge alternent et entrent en interaction avec les éléments internes à l'image qu'ils soulignent, fonctionnant comme un outil de focalisation du regard. Ainsi, l'alternance du bleu et du rouge dans certains bords des cadres permet de distinguer avant-plan et arrière-plan et plus particulièrement espace du sommeil et espace du rêve (f. $40 \mathrm{v}^{\circ}$ ), espace de la mort et espace des vivants $\left(\mathrm{f} .13 \mathrm{v}^{\circ}\right)$, espace de la merveille et espace du monde (f. $35 \mathrm{r}^{\circ}$ ). Elle peut aussi souligner la place qu'occupe le sujet principal dans l'image et le distinguer des personnages de rang inférieur ou secondaires (f. $\left.101 \mathrm{v}^{\circ}\right)^{50}$. À la façon d'une voix surplombant le texte, les marges de l'image fonctionnent comme un narrateur qui organise le regard du lecteur et hiérarchise personnages et espaces.

Si les images de Carpentras sont donc plus attentives au contenu du récit, celles de Paris sont plus soucieuses de sa manière. Elles partagent avec le texte une même pratique : la neutralisation des outils de cadrage traditionnels du regard (formules épiques, liserés de couleur des enluminures)

49 FERlampin-ACHER, Ch. «Épreuves, pièges et plaies dans Artus de Bretagne... », art. cit., p. 167.

50 L'image du f. $40 \mathrm{v}^{\circ}$ montre Artus endormi à qui Proserpine apparaît en songe. La bordure, bleue tout autour du lit, devient rouge dans la partie qui encadre la partie haute dans laquelle se tient le personnage de Proserpine. Le même procédé se retrouve au f. 13v qui illustre la mort de Fenice de la Clere Tor : le bleu encadre le lit de la défunte, le rouge l'espace des vivants qui se tiennent à son chevet. Au f. $35 \mathrm{r}^{\circ}$, on voit Artus fendre en deux un lion monstrueux, enchantement du palais périlleux de la Porte Noire. Le cadre adjacent à l'ouverture dans laquelle est la chambre où se déroule le combat est bleu, et il devient rouge dans sa partie qui entoure le château dont on ne voit que la partie extérieure. Dans notre dernier exemple, au f. $101 \mathrm{r}^{\circ}$, nous voyons un souverain siégeant dans sa tente et recevant un chevalier : la partie adjacente au roi et à son siège est bleue, et souligne sa prééminence, tandis que tout le reste du cadre est rouge. Contrairement à ce qu'indique le site Mandragore (http://visualiseur.bnf.fr/CadresFenetre?O=COMP-1\&I=42\&M=imageseule, page consultée le 28 mai 2014), l'image représente, selon nous, soit le roi Emenidus envoyant son sénéchal porter un message à l'empereur d'Inde, soit ce dernier le recevant, et non « Artus et le roi d'Orcanie ». 
dont le fonctionnement normal est dévoyé. Elles partagent également avec le texte un même goût pour une forme de désinvolture qui réduit le récit à une forme d'insignifiance. Texte et image du manuscrit de Paris se moquent des attentes du lecteur qui se voient déjouées, éventuellement frustrées, car elles n'hésitent pas à souligner les vides du récit, à créer une béance du sens qui est une marque de fabrique d'Artus. De ce point de vue, nous pouvons avancer que le manuscrit de Carpentras, par son souci d'illustrer le roman avec précision, passe à côté de cette esthétique du rajeunissement désinvolte du vieux fonds roulant de la fiction médiévale que le MAîTRE DE FAUVEL a parfaitement compris, ou dont, à tout le moins, l'esthétique iconographique entrait parfaitement en résonance avec celle du roman d'Artus ${ }^{51}$.

\section{Conclusion}

Le rajeunissement du roman entrepris par l'auteur de l'Artus de Bretagne ne manque pas d'ambition. Il s'empare de la matière léguée par ses aînés et leur donne un grand coup de jeune qui affecte le système des personnages, les valeurs romanesques, l'écriture mais aussi, et c'est probablement le plus intéressant, la matérialité de l'un des codex qui nous a transmis la version courte du texte, indice que le fonctionnement désinvolte du texte a été compris des concepteurs du manuscrit qui ont épousé sa vision d'un roman de la fougue et de l'insignifiance.

Ce renouvellement ne passe pas par un rejet de l'héritage littéraire ancien, mais par un courtcircuitage de son fonctionnement premier: les éléments constitutifs d'une topique, littéraire ou iconographique, sont bien présents. Mais ils ne font pas sens comme on s'y attendrait. Ils sont tantôt détournés tantôt neutralisés : les quêtes ne sont pas existentielles, les hypotyposes sont brouillées, les rubriques refusent leur rôle de guide du lecteur et les miniatures s'allègent de détails essentiels au profit de traits stéréotypés.

Dans cet univers où rien ne pèse, la légèreté est une donnée fondamentale et s'accompagne toujours d'un rire de connivence et du plaisir de la reconnaissance. C'est peut-être pour cette raison, et pas seulement par déférence au grand roi de Logres, que le roman a pu être nommé Petit Artus. Mais à trop compter sur son impétuosité et ce que nous avons appelé son énergie citationnelle, le roman s'enferme dans un présent perpétuel et oublie sa fonction mémorielle : négligeant la ramembrance, il perd en senefiance. À force d'être détournée et allégée, la matière romanesque devient une forme vide et prend le risque, une fois évanoui le rire partagé avec les héros du roman, de provoquer chez lecteur le sentiment d'avoir été réellement floué.

51 Précisons en effet que les procédés iconographiques que nous avons relevés ne sont pas propres au roman d'Artus et caractérisent la production du Maître de Fauvel : nous les retrouvons par exemple dans les manuscrits qui nous ont servi de point de comparaison (GuILlaUme DE TYR, Historia, BnF français 22495 et Histoire de Merlin, BnF français 105): focalisation du regard par les liserés bleus et rouges, stéréotypie par utilisation de patrons compositionnels, etc. Nous considérons néanmoins que ces procédés ne prennent sens que dans le cadre d'une interaction avec le texte et que, selon le texte illustré, ce sens se construit différemment, au risque du contresens bien entendu, risque qui nous paraît avoir été plus qu'évité dans le cas du manuscrit de Paris. 